\title{
1 Missing or Underrated Super-emitters of Nitrogen Oxides in China 2 Exposed from Space
}

3 Yuqing Pan ${ }^{1 \#}$, Lei Duan ${ }^{1 \#}$, Mingqi Li ${ }^{1 \#}$, Pinqing Song ${ }^{1 \#}$, Nan Xv ${ }^{1 \#}$, Pengfei $\mathrm{Li}^{1 *}$

$4{ }^{1}$ College of Science and Technology, Hebei Agricultural University, Baoding, Hebei 071000, P.R. China 5

6 "These authors contributed equally to this work.

7

8

9

10 *Correspondence to: Pengfei Li (lpf_zju@163.com); 


\section{Abstract}

19 Nitrogen oxides $\left(\mathrm{NO}_{\mathrm{x}} \equiv \mathrm{NO}+\mathrm{NO}_{2}\right)$ play a central role in air pollution. Super-emitters present unique opportunities for 20 emission mitigation in China and beyond. They comprise intensive industrial facilities (e.g., power or chemical plants), less 21 than $1 \times 1 \mathrm{~km}^{2}$ with high $\mathrm{NO}_{\mathrm{x}}$ plumes, dominating localized concentrations within a limited geographical scope. However, identification of super-emitters typically challenges emission mitigation due to insufficient spatiotemporal resolution. Here we map $\mathrm{NO}_{\mathrm{x}}$ emissions using an efficient, super-resolution $\left(1 \times 1 \mathrm{~km}^{2}\right)$ inverse model based on whole-year TROPOMI satellite observations. Our map offers unique insights on nationwide $\mathrm{NO}_{\mathrm{x}}$ super-emitters. We resolve 1625 super-emitters in virtually every corner in China, even in remote and mountainous zones, which we trace back to either an industrial hotspot or a cluster (i.e., an industrial park). The state-of-the-art bottom-up emission inventory MEICv1.3 largely (67\%) agrees with our results within a factor of two for cities. However, that inventory does not identify super-emitters, particularly underestimating one-third of the emissions by at least an order of magnitude. Many individual industrial hotspots are often found to be displaced or missing. Moreover, traditional top-down inverse methods do not effectively detect such superemitters. Here we show it is necessary to address the $\mathrm{NO}_{\mathrm{x}}$ budget by revisiting super-emitters on a large scale. Integrating the results we obtain here with a multi-tiered observation system can lead to identification and mitigation of anomalous $\mathrm{NO}_{\mathrm{x}}$ emissions.

\section{Introduction}

34 Nitrogen oxides $\left(\mathrm{NO}_{\mathrm{x}} \equiv \mathrm{NO}+\mathrm{NO}_{2}\right)$ play a central role in the formation of fine particular matter and ozone and have implications for climate change, human health, and life expectancy ${ }^{1-4}$. They are typically emitted by all combustion processes, particularly industrial activities (e.g., power or chemical plants) $)^{5-8}$. China is a case in point. There is a heavy-tail distribution of $\mathrm{NO}_{\mathrm{x}}$ emission sources in a number of localized regions ${ }^{9,10}$, where a few sources (so-called super-emitters) generally emit highly concentrated $\mathrm{NO}_{\mathrm{x}}$ plumes and might even dominate localized emissions with limited geographical extent (i.e., $\sim 1 \times 1 \mathrm{~km}^{2}$ ). Hence, unique opportunities for mitigation are presented by super-emitters, particularly by those due to leaks and abnormal operating conditions ${ }^{11,12}$.

41 However, efforts to guide super-emitter mitigation are complicated by large inconsistencies between emission estimates ${ }^{13-16}$.

42 This is primarily because these inventories are generally designed at the regional scale rather than localized super-emitters.

43 For instance, the foundations of bottom-up inventories, such as activity data and emission factors, are often outdated, sparse, and unrepresentative for super-emitters ${ }^{5,7,17,18}$. By comparison, top-down attempts relying on relatively accurate and up-todate measurements present a more promising future ${ }^{6}$. Nevertheless, there is a dearth of available regular measurements for the super-emitters ${ }^{19}$. Field campaigns are also spatially sparse and temporally infrequent, thus inapplicable for the superemitters distributed over a large scale. 
For decades, satellite measurements have provided spatial patterns and magnitude of tropospheric $\mathrm{NO}_{2}$ vertical column densities (VCDs) on a global scale, central to improving our knowledge of the $\mathrm{NO}_{\mathrm{x}}$ emission budget ${ }^{1,6,20}$. However, the ability to detect super-emitters has been limited by pixels far larger than $1 \times 1 \mathrm{~km}^{221}$. Finally, the emissions attributed to the $\mathrm{NO}_{\mathrm{x}}$ super-emitters have not been well isolated and evaluated nationwide.

The TROPOspheric Monitoring Instrument (TROPOMI) on the Sentinel-5 Precursor has an unprecedented spatial resolution of up to $3.5 \times 7 \mathrm{~km}^{2}\left(3.5 \times 5.5 \mathrm{~km}^{2}\right.$ from August 2019 onward $)$ and a high signal-to-noise ratio ${ }^{7,13}$. A representative topdown method using a CTM and a Kalman Filter has tested the TROPOMI measurements at a sacrifice of spatial resolution (i.e., $\left.\sim 0.25^{\circ} \times 0.25^{\circ}\right)^{22}$, consequently focusing only on regional issues. A recent study preserved the benefit of its high spatial resolution and experimentally exposed a few large emission sources on a national scale ${ }^{7}$. However, the original TROPOMI's resolution $\left(3.5 \times 5.5 \mathrm{~km}^{2}\right)$ still has a crucial gap in the scale of the localized super-emitters $\left(\sim 1 \times 1 \mathrm{~km}^{2}\right)$. Here we develop an efficient, super-resolution (i.e., from $3.5 \times 5.5 \mathrm{~km}^{2}$ to $1 \times 1 \mathrm{~km}^{2}$ ) inverse model by capitalizing on a whole year of daily TROPOMI measurements (Methods). The retrievals of the $\mathrm{NO}_{\mathrm{x}}$ VCDs are supported by a state-of-the-art CTM (i.e., the WRF-CMAQ model). To prevent the super-emitters from being omitted, a key advance of this model is to take into account the nonlinear $\mathrm{NO}_{\mathrm{x}} \mathrm{VCD}$-Transport-Lifetime-Emission relationships and to integrate an oversampling $\operatorname{method}^{7,14}$. The objective is to provide the first systematic survey of the $\mathrm{NO}_{\mathrm{x}}$ emission distributions at the super-resolution, with a focus on geo-locating and quantifying the super-emitters nationwide, down to the industrial hotspots or parks. Our survey is used to benchmark a state-of-the-art emission inventory MEICv1.3 (the Multi-resolution Emission Inventory for China) and also a state-of-the-art top-down product. This will help fill an important gap in scale and re-evaluate the $\mathrm{NO}_{\mathrm{x}}$ budget and hotspots in China.

\section{Identification of super-emitters}

An oversampling approach was applied to exploit the variable spatial coverage of the satellite pixels (Methods). As a result, we achieved the super-resolution (i.e., from $3.5 \times 5.5 \mathrm{~km}^{2}$ to $1 \times 1 \mathrm{~km}^{2}$ ) tropospheric $\mathrm{NO}_{\mathrm{x}}$ VCDs based on the TROPOMI measurements in China (Fig. 1a). We also zoomed in on five sub-regions (including Northwest, Northeast, North, Southwest, East, and South China) (Figs. $1 \mathrm{~b} \sim 1 \mathrm{~g}$ ). On this basis, the consequent $\mathrm{NO}_{\mathrm{x}}$ emission map was derived by an efficient, compatible top-down inverse model (Fig. S1). In theory, this set of maps (Fig. 1 and Fig. S1) is distinct from previous outcomes obtained from early satellite-based surveys (e.g., those based on the OMI or TROPOMI measurements) ${ }^{13,14,23}$. This is solely attributed to major improvements in the model developed here, which is of hyperfine resolution and takes meteorological and chemical effects into account (Methods; Supplementary Information). An illustrative comparison is explained in the next section. We isolated and identified 1625 hotspots (Fig. 1) that are inventoried in Supplementary Table 71 only if they could be resolved unambiguously on the basis of the maps alone without the need for a priori knowledge. Each 8 exhibited a prominent localized (i.e., $1 \times 1 \mathrm{~km}^{2}$ ) $\mathrm{NO}_{\mathrm{x}}$ enhancement regarding both VCDs and emissions. 
79 Spatially, we found that these super-emitters are scattered on every corner in China, even in remote zones close to the

80 frontiers (e.g., Northwest and Southwest China) (Fig. 1). As expected, such super-emitters concentrated over North, East,

81 and South China. By combining the Landsat 8 images, the identified super-emitters can be classified into two classes: industrial hotspots and industrial parks. They can be linked to the super-emitters typically containing individual point sources or intensive industrial facilities. Illustrative examples are shown in Fig. 2 and Fig. S3, and detailed spatial information is highlighted in Fig. S2. The 936 hotspots in the class of industrial hotspots were consistently found to be associated with isolated industrial factories or power plants with one or more chimneys, as presented in the visible images. For instance, a typical localized $\mathrm{NO}_{\mathrm{x}}$ emission maximum was found in a remote desert area in Northwest China (Fig. 2a), coinciding spatially with a large industrial factory (Zeketai, Northwest China), as shown in the satellite image. It was adjacent to a small city (i.e., Xinyuan in Xinjiang Province) dominated by residential areas, but with much lower $\mathrm{NO}_{\mathrm{x}}$ distributions and emissions. Longzhudou (East China) (Fig. 2b) and Huaxin (Southwest China) (Fig. S3a) were similar examples of industrial hotspots. They were also planted in mountainous areas but close to medium-sized cities (Ningde in Fujian Province and Lhasa in Tibet). The Jincheng Steel Group, located in North China, is a key base of the iron and steel industry (Fig. 2c). $\mathrm{NO}_{\mathrm{x}}$ emissions associated with coal-fired power plants were also identified, for example, in Rundajianeng (Northwest China) (Fig. 2d) and Mudanjiang (Northeast China) (Fig. S3b).

The second class, that of industrial parks, is linked to aggregative zones with massive industrial facilities but limited geographical extent (i.e., $1 \times 1 \mathrm{~km}^{2}$ ), for which nearly 700 parks were detected. They were mostly associated with chemistry and manufacturing industries, such as oil and gas, iron and steel, and foundry production. Well-isolated examples include the parks in Baitong (Northwest China) (Fig. 2e), Shizuishan (North China) (Fig. 2f) Zhongluan (North China) (Fig. S3c), and Beiying (Northeast China) (Fig. S3d). These super-emitters were clearly detected in the set of the maps, despite already large background concentrations. Chemical industrial parks, due to their large production, were often found to be geographically close to their distribution market. Nanbao (East China) (Fig. 2g) and Jiaochuan (East China) (Fig. S3e) are the representative and bordered on the Beijing-Tianjin-Hebei region and the Yangtze River Delta region, respectively. Many industrial parks were also found near raw-material-related industries (e.g., oil fields and coal mines), thus conducive to building an agile supply chain. Daqing (Northeast China) (Fig. 2h) is an archetype of such a super-emitter, which is next to the famous Daqing Oilfield and railway stations. Moreover, such examples are abundant. For instance, the large petrochemical industrial parks, such as Longmen (Northwest China) (Fig. S3f) and Shengbang (Northwest China) (Fig. S3g), were also close to the coal bases (i.e., the Hancheng Coal Mine and the Shenmu Coal Mine).

Commonly, rural regions were also found with enhanced $\mathrm{NO}_{\mathrm{x}} \mathrm{VCDs}$ and emissions. These regions correspond to, for example, residential areas and small-scale manufacture clusters, such as Jiqingbao (North China) (Fig. S4a), Pangjing (East China) (Fig. S4b), and Chetian (Southwest China) (Fig. S4c). The primary $\mathrm{NO}_{\mathrm{x}}$ sources might be attributed to scattered coal combustion $^{24,25}$. Nevertheless, it was difficult to link those sources with clear, well-isolated super-emitters. Also, $\mathrm{NO}_{\mathbf{x}}$ emissions in cities represent a substantial part of the total atmospheric $\mathrm{NO}_{\mathrm{x}}$ budget. Megacities, such as Beijing (Fig. S4d), 
112 Shanghai (Fig. S4e), Guangzhou (Fig. S4f), and Shenzhen (Fig. S4g), are the cases in point. We emphasized that, from a

113 narrower spatial perspective (i.e., $\sim 10 \times 10 \mathrm{~km}^{2}$ ), small-medium cities, such as Lhasa (Southwest China) (Fig. S4h),

114 Shizuishan (Northwest China) (Fig. S4i), and Yongan (South China) (Fig. S4j), are no exception. The main sources can be

115 consistently related to urban transportation, which, however, are too diffuse to emerge as individual super-emitters in our

116 maps. In addition, the one-year average of satellite detections was generally difficult to capture discontinuous and

117 instantaneous biomass burning. Thus, hotspots dominated by biomass burning were excluded from this study.

\section{Assessment of super-emitters}

119 We have compared the emission fluxes from all the super-emitters and 370 cities in China in our model with those in the 120 state-of-the-art bottom-up emission inventory MEICv1.3 (Methods, Supplementary Information) (Supplementary Table 1).

121 For $67 \%$ of the cities, the emission fluxes agreed within a factor of two (85\% within a factor of three) and, importantly, when

122 all large and medium-sized cities were considered, no major bias emerged. Note that, compared to our results, the inventory 123 often underestimated the $\mathrm{NO}_{x}$ emissions in remote small-sized cities significantly (by more than a factor of five)

124 (Supplementary Table 1). Therein some isolated super-emitters (i.e., industrial hotspots or parks), such as Hejing (Northwest 125 China), Geermu (Northwest China), and Luopu (Northwest China), were directly missed (Figs. S5a S5c). It should be 126 emphasized that, in turn, our estimates of $\mathrm{NO}_{\mathrm{x}}$ emissions in several megacities, such as Shanghai and Shenzhen, are less than 127 those in MEICv1.3 to some extent (i.e., > -20\%). This could be attributed to persistent air pollution controls in China since $1282013^{26}$. In contrast, it was immediately clear that the emission fluxes of the super-emitters were largely underestimated in 129 MEICv1.3, irrespective of their classes. Of all the identified super-emitters, only 35\% agreed within a factor of three and, 130 more importantly, only $72 \%$ within one order of magnitude. Overall, they exhibit a heavy-tail distribution, indicating that $20 \%$ 131 of the identified hotspots were responsible for $40 \%$ of all the detected super emissions (Fig. 3 and Supplementary Table 1).

132 Compared to MEICv1.3, this satellite survey reveals three kinds of super-emitters. First, 460 super-emitters are identified 133 owing to a nearby localized maximum in the bottom-up inventory, despite exceptionally low emission fluxes. Representative 134 examples were presented in Fig. 3, including Maligou (Northwest China) (Fig. S5d) for an industrial hotspot and Sanbaotun 135 (Northeast China) (Fig. S5e) and Peijiafen (North China) (Fig. S5f) for two industrial parks.

136 Second, compared to MECIv1.3, nearly 300 super-emitters seemed displaced in various degree, by at least one grid cell in 137 MEICv1.3 (i.e., $25 \times 25 \mathrm{~km}^{2}$ ) (from the identified super-emitter centre), for example, Zhongjing (Southwest China) (Fig. 138 S5g) and Guolemude (Northwest China) (Fig. S5h). Third, the other super-emitters did not represent a localized maximum in 139 MEICv1.3 and were substantially underestimated compared to our results. They are essentially absent from the inventory 140 because their fluxes are at least one order of magnitude lower than our surveyed results. Notably, Nanzamu (Northeast China) 141 (Fig. S5i), Chengbei (Northwest China) (Fig. S5j), Tanjiazui (South China) (Fig. S5k), and Mengsheng (Southwest China) 142 (Fig. S51) were captured by the one-year satellite observations. They represented the localized (i.e., $1 \sim 10 \mathrm{~km}$ ) maximum but 143 were undoubtedly missed in the inventory. 
144 Besides, the satellite-based study could capture the onset or the discontinuation of industrial activities unambiguously. New

145

146

148

149

150

151

152

153

154

155

156

157

158

159

160

161

162

163

164

165

166

167

168

169

170

171

172

173

174

175

or expanded super-emitters that emerged within the satellite measurements were found in this way. Correspondingly, the satellite-based emission fluxes were significantly higher (at least one order) than the bottom-up estimations. For instance, high $\mathrm{NO}_{\mathrm{x}}$ emissions were observed over Xincheng (North China) (Fig. S6a) and Huagang (South China) (Fig. S6b). On the other hand, industrial plant closures were also detected, for example, over Shidian (North China) (Fig. S6c) and over Hunhe (Northeast China) (Fig. S6d). Therein the bottom-up emission inventory was likely outdated, overestimating the satellitebased estimates by more than $100 \%$.

Figure 4 shows the stable monthly variations (i.e., $<26 \%$ ) in the satellite-based emission estimates of eight representative super-emitters. The detailed variation information is shown in Fig. S7. Therefore, for most of the super-emitters, one-month satellite overpasses could derive their yearly emission estimates and be capable of tracking their emission variations.

\section{Discussion}

Here we develop an efficient, super-resolution inverse approach by collecting an entire year of daily TROPOMI measurements, applying an oversampling method, and building a $\mathrm{NO}_{\mathrm{x}} \mathrm{VCD}$-Transport-Lifetime-Emission model. The key for preventing the smearing of the super-emitters is to exploit the super-resolution and wind-driven horizontal fluxes. This is particularly useful for preserving strong gradients close to super-emitters even on top of considerably high pollution. In contrast, current top-down approaches generally apply time-consuming inverse algorithms to satellite measurements, thus sacrificing spatial resolution and focusing on regional issues ${ }^{29}$. We compared our results with a state-of-the-art top-down $\mathrm{NO}_{\mathrm{x}}$ emission inventory ${ }^{22}$ for three representative super-emitters (Fig. S8). Although this inventory also relied on the TROPOMI observations, it adopted another inverse model (i.e., the DESCO algorithm) to explore regional emission variations on a $0.25^{\circ} \times 0.25^{\circ}$ resolution. Figures S8 and S9 demonstrate that these two products were very similar in the general spatial distributions and regional magnitude. In contrast, a key advance in this study was the considerable increase in spatial resolution. Of particular relevance are the three super-emitters, which can only be distinguished in our results.

This work has presented a detailed and hyperfine inventory of $\mathrm{NO}_{\mathrm{x}}$ super-emitters over China. They can be consistently linked to either industrial hotspots or parks and responsible for the localized $\mathrm{NO}_{\mathrm{x}}$ budget. More importantly, their emissions are mostly underestimated, even displaced and missed, in a widely used emission inventory. This work can also capture the emergence or closure of super-emitters in a relatively short time (i.e., monthly). Note that manual efforts regarding distinguishing the super-emitters limit this study. Deep learning algorithms might be a prospective alternative that allows us to rapidly and impartially identify super-emitters ${ }^{30}$. To date, continuous emission monitoring systems (CEMS) remain largely absent for super-emitters, particularly in industrial parks. Our results suggest that it is necessary to revisit traditional bottom-up $\mathrm{NO}_{\mathrm{x}}$ inventories. Otherwise, it is quite possible to mislead local air pollution distributions in CTMs and thus local efforts for mitigation. By comparison, satellite surveys can make an important contribution to monitoring $\mathrm{NO}_{\mathrm{x}}$ emissions, particularly beneficial for up-to-date emission inventories for quickly developing countries. Therefore, widespread and 
176 sustained deployment of a multi-tiered observational strategy, i.e., a combination of this hotspot detection technique with a 177 near-real-time ground-based and another satellite-based monitoring network of regional sources, could greatly advance 178 scientific understanding of $\mathrm{NO}_{\mathrm{x}}$ budgets. More specifically, our approach, together with more comprehensive bottom-up 179 information, could be expanded to detect abnormal (e.g., leakage) facilities ${ }^{31}$ on a large spatial scale. More hyperfine 180 satellite-based measurements (e.g., GHGsat for methane, $\left.\sim 50 \times 50 \mathrm{~m}^{2}\right)^{32,33}$ might further facilitate the isolation and 181 identification of the super-emitters in intensive source regions. 
Submitted to Nature Communication
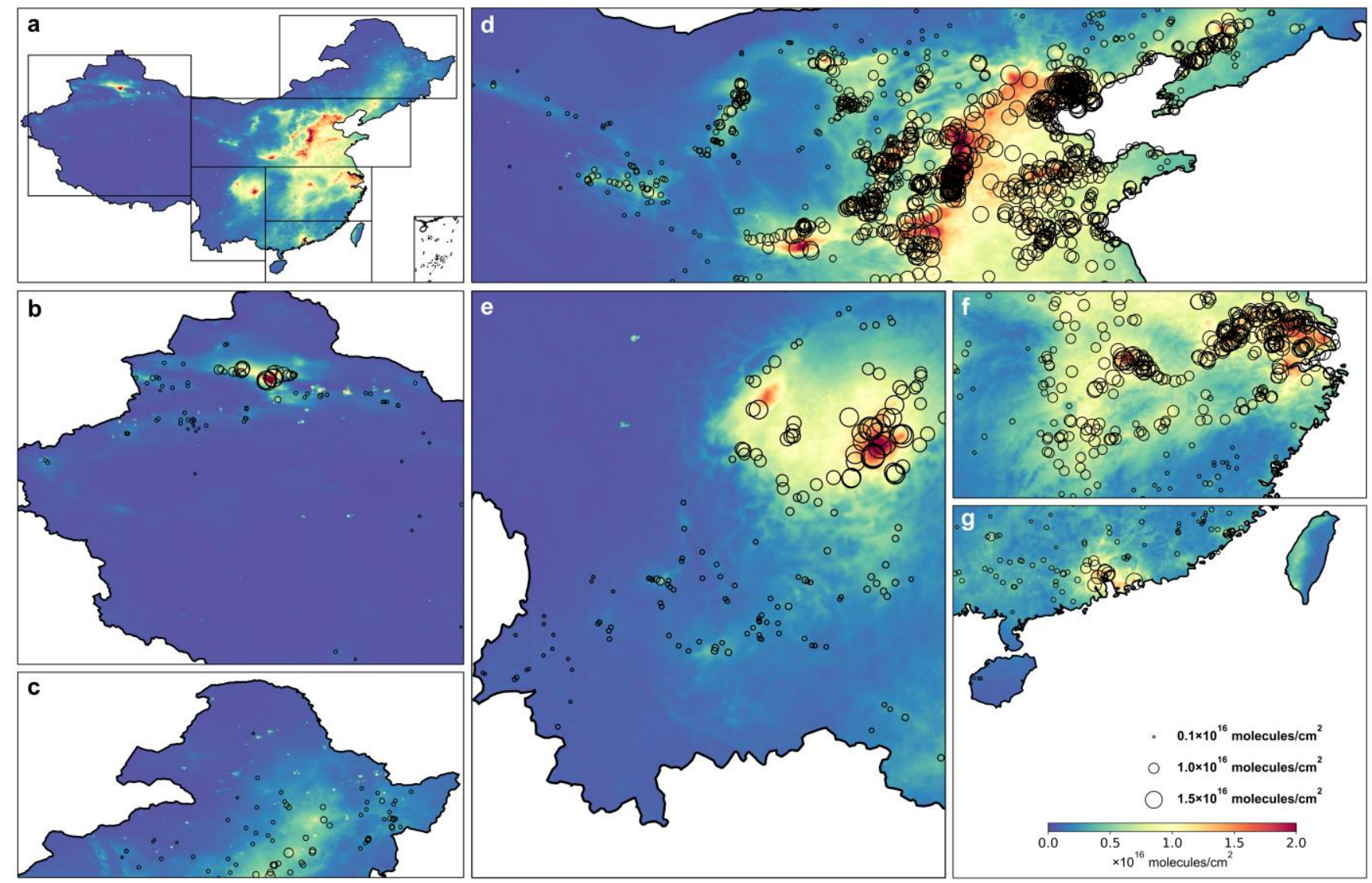

Fig. 1. One-year oversampled NOx VCDs and emission hotspots based on the TROPOMI instrument. a, One-year oversampled $\mathrm{NO}_{\mathrm{x}}$ VCDs across China. b g, Zoom-ins over Northwest, Northeast, North, Southwest, East, and South China, which were marked by the black rectangles in Fig. 1a. Super-emitters are marked by black circles and scattered over China; their sizes denote the satellite-based emission fluxes. Detailed information of the super-emitters are recorded in Supplementary Tables 1 and 2 and Supplementary Map of the Super-emitters. 
Submitted to Nature Communication
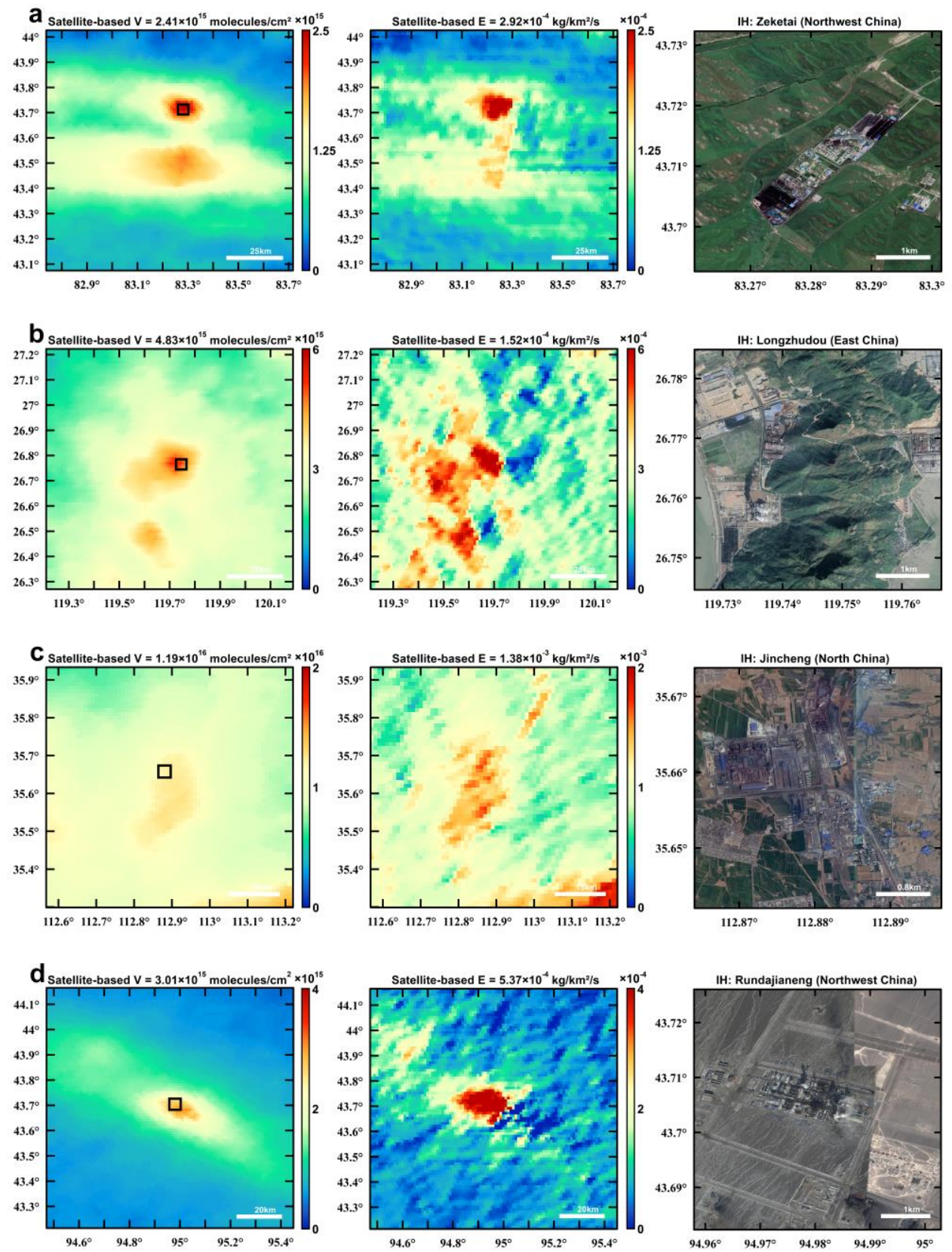

Fig. 2. Illustrative examples of super-emitters, including industrial hotspots (IH) and parks (IP). a h, For each site,

192 the left and medium panels offer the TROPOMI-based $\mathrm{NO}_{\mathrm{x}}$ VCDs and emissions, respectively. The vertical and horizontal

193 axes correspond to latitude and longitude, respectively. The right panels offer a close-up view of the super-emitters outlined

194 by the black rectangles in the left panel. Satellite-based V and E denote the satellite-based VCDs and emissions, respectively.

195 The satellite images come from the Landsat 8 imageries (Fig. S2a $\sim$ S2d). 
Submitted to Nature Communication
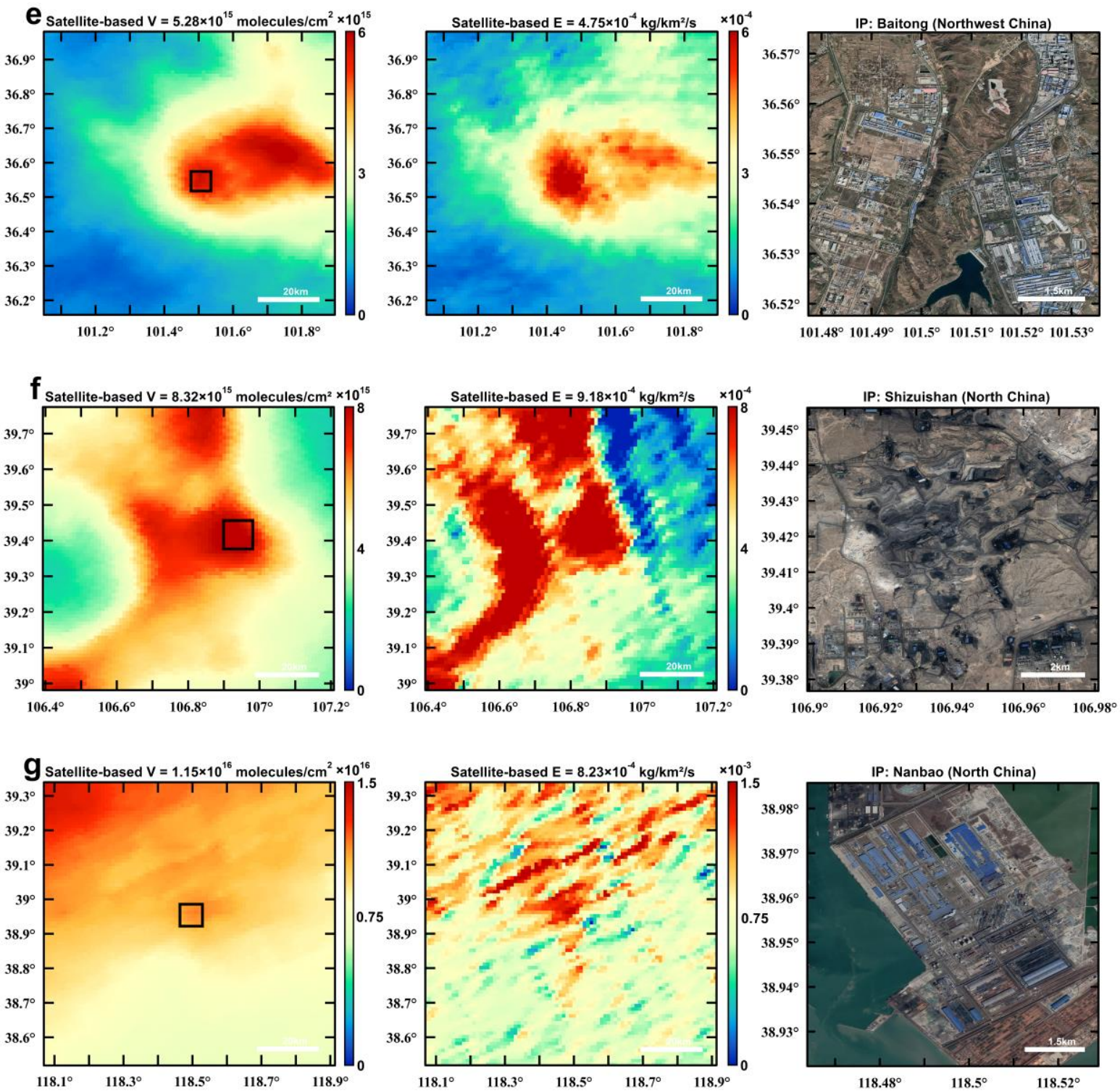

h Satellite-based $\mathrm{V}=3.82 \times 10^{15} \mathrm{molecules} / \mathrm{cm}^{2} \times 10^{15}$
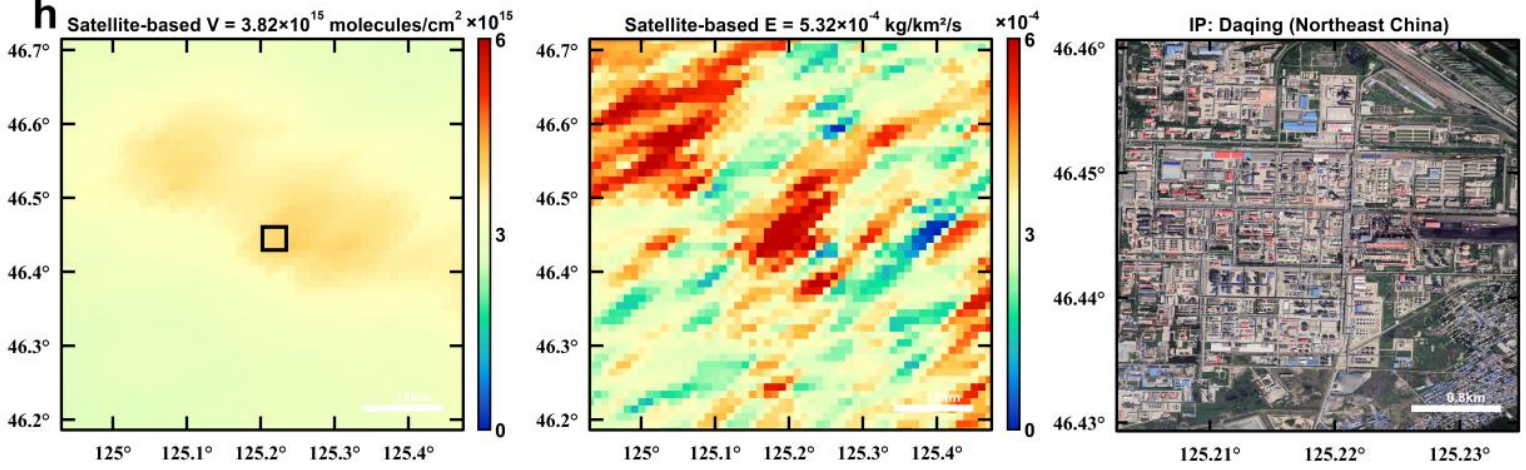
197 Fig. 2. Illustrative examples of super-emitters, including industrial hotspots (IH) and parks (IP). a h, For each site,

198 the left and medium panels offer the TROPOMI-based $\mathrm{NO}_{x}$ VCDs and emissions, respectively. The vertical and horizontal

199 axes correspond to latitude and longitude, respectively. The right panels offer a close-up view of the super-emitters outlined

200 by the black rectangles in the left panel. Satellite-based V and E denote the satellite-based VCDs and emissions, respectively.

201 The satellite images come from the Landsat 8 imageries (Fig. S2e $\sim$ S2h). 
Submitted to Nature Communication

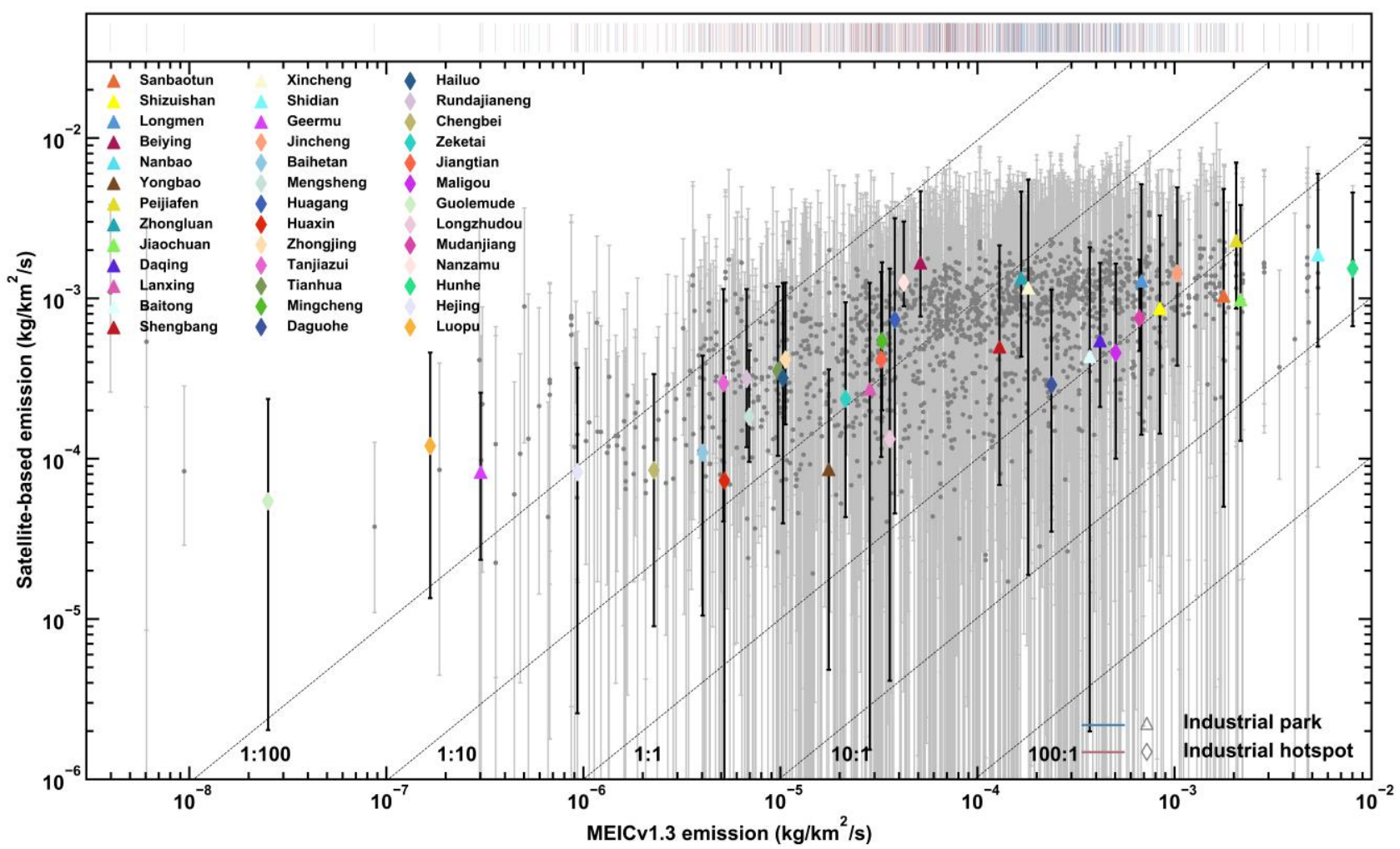

203 Fig. 3. Satellite-derived $\mathrm{NO}_{\mathbf{x}}$ emission fluxes versus a bottom-up emission inventory (MEICv1.3). Satellite-based $\mathrm{NO}_{\mathrm{x}}$

204 emission estimates for the cities (diamonds) and super-emitters, including industrial hotspots (triangles) and parks (circles)

205 versus bottom-up $\mathrm{NO}_{\mathrm{x}}$ emission estimates in MEICv1.3. To track the cities and super-emitters more clearly, we successively

206 each one by a vertical line in the top panel. The five dashed oblique lines from left to right represent ratios of the MEIC-

207 based emissions to the satellite-based emissions of 1:100, 1:10, 1:1, 10:1, and 100:1. The satellite-based emission fluxes are

208 calculated assuming a baseline $\mathrm{NO}_{\mathrm{x}}$ lifetime of 4 hours (Methods). The error bars correspond to upper- and lower-bound flux

209 estimates based on a lifetime of 1 hour and 24 hours, respectively. Biomass-burning hotspots are omitted from this

210 comparison, which are not included in MEICv1.3. 
Submitted to Nature Communication

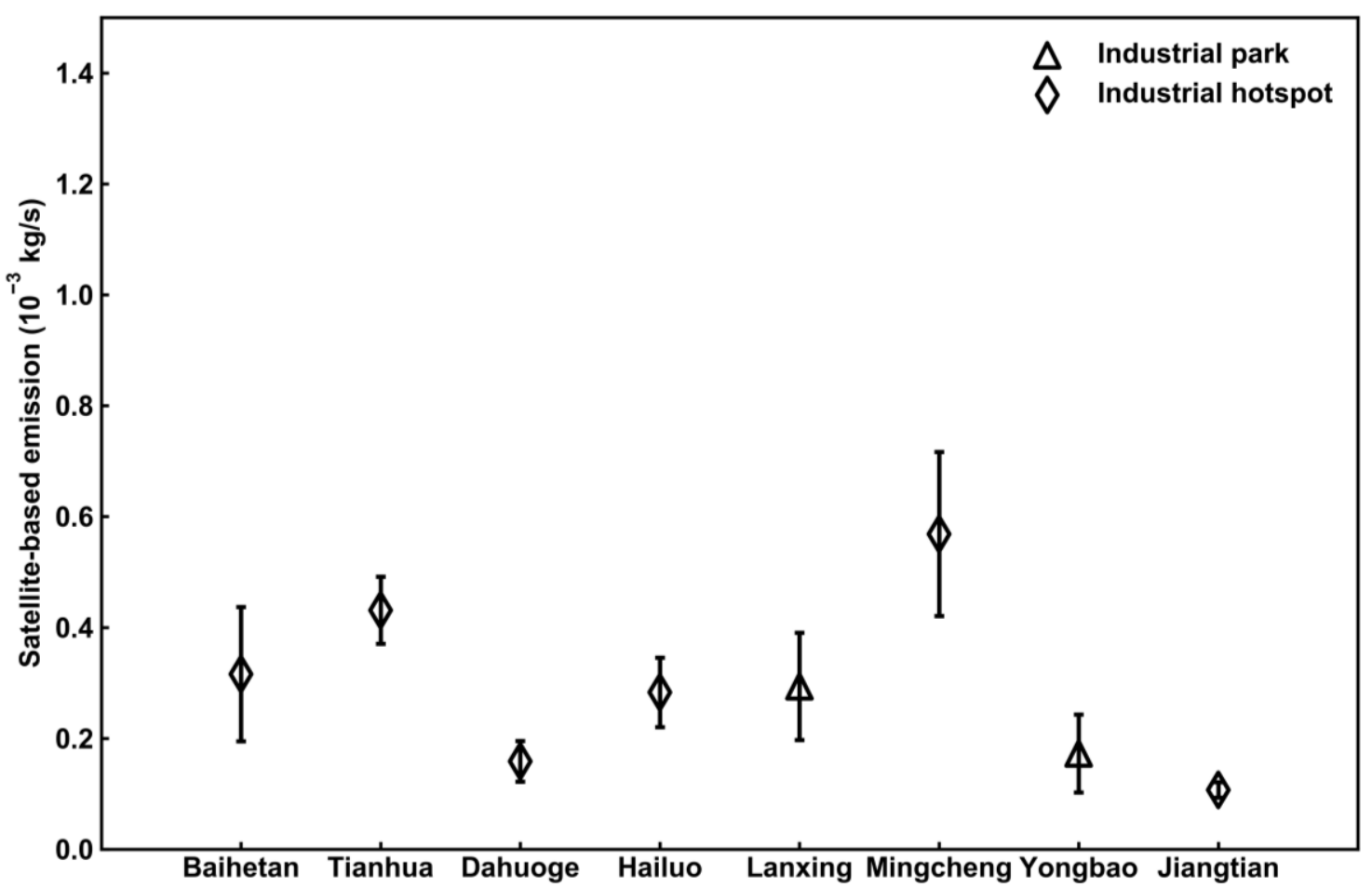

213 Fig. 4. Representative examples of super-emitters that show stable monthly variations in the satellite-based NOx

214 emissions. The error bars represent $1-\sigma$ uncertainties. 


\section{Satellite observations}

218 The TROPOMI instrument on the European Space Agency's Sentinel-5P satellite provides daily global coverage of slant 219 column densities ( $\mathrm{SCDs})$ of $\mathrm{NO}_{2}$ with an unprecedented spatial resolution of up to $3.5 \times 7 \mathrm{~km}^{2}\left(3.5 \times 5.5 \mathrm{~km}^{2}\right.$ from August 2202019 onward $)^{7,13,34}$. Its overpass time is close to noon (13:30 local time). The satellite measurements with a high resolution 221 allow us to analyze the finer scale spatiotemporal characteristics of $\mathrm{NO}_{2}$. On this basis, the following calculation of 222 tropospheric $\mathrm{NO}_{2}$ VCDs was very similar to the operational product ${ }^{35}$, for which detailed algorithms can be found in the

223 production discription ${ }^{36}$. However, the difference was associated with the conversion of SCDs to VCDs that relied on the air mass factor (AMF) approach. In the operational product, the a priori $\mathrm{NO}_{2}$ profile was taken from the global chemistry transport Tracer Model 5 (TM5-MP) with a spatial resolution of $1^{\circ} \times 1^{\circ}$. By comparison, we improved this process by using a priori profile from a comparably high resolution (i.e., $5.5 \times 5.5 \mathrm{~km}^{2}$ ) CTM (i.e., the WRF-CMAQ model ${ }^{37,38}$ ). Note that measurements with cloud fraction above $30 \%$ or a "qa value" (indicating data quality) below 0.75 were skipped ${ }^{36}$. For each pixel, to derive the lower VCD and eliminate the bias of the stratospheric estimate, we subtracted the $5^{\text {th }}$ percentile within the individual pixel from the total $\mathrm{VCD}^{7}$.

\section{The WRF-CMAQ model}

The two-way coupled WRF-CMAQ model (the WRF-CMAQ model) was applied to provide a priori profile and wind fields on the spatial resolution of $5.5 \times 5.5 \mathrm{~km}^{2}$ (comparable to the resolution of the TROPOMI instrument). The results are the foundation of the AMF calculation and the following top-down $\mathrm{NO}_{\mathrm{x}}$ emission estimates. Detailed model settings can be found in our previous papers ${ }^{39,40}$. Meteorological initial and boundary conditions were obtained from the European Centre for Medium-range Weather Forecasts (ECMWF) ERA5 reanalysis dataset with the spatial resolution of $1^{\circ} \times 1^{\circ}$ and temporal resolution of 6 hours. The analysis nudging option was switched on for temperature, humidity above the PBL, and winds at all model levels, thus being nudged to the meteorological driving data (i.e., the ERA5 reanalysis dataset) ${ }^{41}$.

The horizontal domain of the model covered mainland China with a compatible horizontal resolution $\left(5.5 \times 5.5 \mathrm{~km}^{2}\right)$ following a Lambert Conformal Conic projection (Fig. 1a). In terms of the vertical configuration, 29 sigma-pressure layers ranged from the surface to the upper-level pressure of $100 \mathrm{hPa}, 20$ layers of which are located below around $3 \mathrm{~km}$ to derive finer meteorological and chemical characteristics within the planetary boundary layer.

The anthropogenic emissions were obtained from MEICv1.3 ${ }^{15}$, which contained primary species (e.g., primary $\mathrm{PM}_{2.5}, \mathrm{SO}_{2}$, $\mathrm{NO}_{\mathrm{x}}, \mathrm{CO}$, and $\mathrm{NH}_{3}$ ) from five anthropogenic sectors (i.e., agriculture, power plant, industry, residential, and transportation). This inventory was initially designed with the spatial resolution of $0.25^{\circ} \times 0.25^{\circ}$ and thus reallocated to match the domain configuration (i.e., $5.5 \times 5.5 \mathrm{~km}^{2}$ ) in the study. 
247 To achieve a super-resolution reconstruction for the one-year tropospheric $\mathrm{NO}_{2}$ VCDs, we applied the oversampling method 248 to convert from the original satellite pixels to the $1 \times 1 \mathrm{~km}^{2}$ grid cells. As demonstrated in previous studies associated with 249 reshaping air pollutant distributions, this technique can exploit the fact that the location, shape, and orientation of the satellite footprints slightly varies from one orbit to another ${ }^{14,18}$. Thus, a much higher resolution can be obtained on the spatial distribution by sacrificing temporal information. This was distinct from the most widely used geometry methods that generally rely on interpolation or binning on a rectangular latitude-longitude grid ${ }^{42}$.

Here the oversampling technique we adopted was similar to early attempts ${ }^{18,42,43}$. Initially, a grid size of $1 \times 1 \mathrm{~km}^{2}$ was chosen. For each overpass, the TROPOMI footprint pixel coverage was calculated and, for computational reasons, approximated as an ellipse on a rectangular latitude-longitude grid. And then, we calculated the area-averaged value using all measurements overpassing the given cell. During this process, we also considered the influences of the footprint size that was set as a weight inversely proportional to the area of each footprint. Nevertheless, a measurement result would be regarded as erroneous values and thus eliminated when it was more than 10 standard deviations from the average ${ }^{18}$. As a result, $3.12 \%$ of the measurements were eliminated in this way.

\section{The top-down $\mathrm{NO}_{\mathrm{x}}$ emission model}

We developed an efficient, super-resolution (i.e., $1 \times 1 \mathrm{~km}^{2}$ ) top-down inverse model on the basis of the one-year oversampled TROPOMI $\mathrm{NO}_{2}$ VCDs. This model took into account their chemical and meteorological loss, including chemical loss and horizontal transport. Therein the horizontal fluxes considered both time-averaged advection and diffusion processes $^{7}$, while vertical fluxes, either upward or downward transport, were neglected, owing to their small contributions to the $\mathrm{NO}_{\mathrm{x}}$ budget $^{14}$. The latter assumption was used in numerous studies ${ }^{7,14}$. This model was assumed to be equilibrium $\left(\frac{\partial V_{i, j}}{\partial t}=\right.$ 0), the governing equation of which was thus shown as follows (Eqs. $1 \sim 4$ ):

$\frac{\partial V_{i, j}}{\partial t}=r E_{i, j}-\frac{V_{i, j}}{\tau_{i, j}}-w_{i, j} \cdot \nabla V_{i, j}+\nabla \cdot\left(k_{i, j} \cdot \nabla V_{i, j}\right)=0$ (Eq. 1).

Therein $\boldsymbol{V}_{\boldsymbol{i}, \boldsymbol{j}}$ represents the tropospheric $\mathrm{NO}_{2} \operatorname{VCDs}$ in the grid $(\boldsymbol{i}, \boldsymbol{j})$ of $1 \times 1 \mathrm{~km}^{2} . \boldsymbol{E}_{\boldsymbol{i}, \boldsymbol{j}}$ denotes all ground $\mathrm{NO}_{\mathrm{x}}$ sources, which combines anthropogenic, soil, and biomass burning $\mathrm{NO}_{\mathrm{x}}$ emissions. $\boldsymbol{r}$ represents the ratio of $\mathrm{NO}_{2}$ over $\mathrm{NO}_{\mathrm{x}}$ concentration. In theory, the daytime $\mathrm{NO}_{x}$ chemical system reaches equilibrium rapidly and $\boldsymbol{r}$ varies little. In this study, we set $\boldsymbol{r}$ to be $0.76^{7,14}$. The remaining terms in Eq. 1 represent the potential $\mathrm{NO}_{\mathrm{x}}$ sinks, including chemical, deposition, horizontal, and diffusional loss. Given that the local overpass time of TROPOMI is close to noon (13:30 local time), the chemical $\mathrm{NO}_{\mathrm{x}}$ sink is dominated by the chemical loss reaction of $\mathrm{NO}_{2}$ with $\mathrm{OH}$, which can be described by a first-order time constant $\boldsymbol{\tau}_{i, j}$ and, thus, can be estimated from the measured $\boldsymbol{V}_{i, j}$ itself as $\frac{\boldsymbol{V}_{i, j}}{\boldsymbol{\tau}_{i, j}}$. $\boldsymbol{\tau}_{\boldsymbol{i}, j}$ indicates the lifetimes associated with deposition and chemical loss in nature. In theory, instantaneous $\mathrm{NO}_{\mathrm{x}}$ lifetime is dominated by several factors, such as ozone levels and actinic fluxes, and 
also the $\mathrm{NO}_{\mathrm{x}}$ concentration itself at the presence of high $\mathrm{NO}_{\mathrm{x}}$ levels. As a result, the $\mathrm{NO}_{\mathrm{x}}$ lifetime is linked with season and meteorological conditions. Therefore, the assumption of the $\mathrm{NO}_{\mathrm{x}}$ lifetime is a major limitation of this method and might be inappropriate for super-emitters ${ }^{6,7,14,44}$.

However, previous studies have demonstrated that the assumed lifetime could appropriately relate the measured $\boldsymbol{V}_{\boldsymbol{i}, \boldsymbol{j}}$ to the actual emissions $\boldsymbol{E}_{\boldsymbol{i}, \boldsymbol{j}}$ via mass balance. As shown in previous attempts in Riyadh, the $\mathrm{NO}_{\mathrm{x}}$ lifetime has been derived to 4 hours with an uncertainty of $35 \%$. Its seasonal variations were found to be weak. In the United States and China, a similar method was applied to cities and power plants, resulting in a mean lifetime of $3.8 \pm 1.0$ hours for May-September ${ }^{23}$. In this study, we utilized a value of 4 hours for all regions. For many of the hotspots, there can be substantial transport out of the box, in which case the effective lifetime would be smaller than 4 hours. Given the high spatial resolution, the emission estimates of a grid cell derived in this way were more likely to be underestimated than overestimated. For specific remote sites, however, 4 hours could be too short.

Therefore, we applied conservative assumptions using lifetimes of 1 hour and 24 hours to provide upper- and lower-bound emission estimates. The corresponding uncertainties are considered and shown as error bars in Fig. 3. Although more sophisticated methods, like regional CTM simulations, are available, they would not significantly optimize the superresolution emission estimates for the super-emitters. Alternatively, we could further introduce satellite-based HCHO VCDs into our framework to constrain the $\mathrm{OH}$ distributions ${ }^{45}$, which might be useful for optimizing the model.

$\boldsymbol{w}_{i, j} \cdot \boldsymbol{\nabla} \boldsymbol{V}_{\boldsymbol{i}, j}$ denotes the time-averaged advection term. Therein $\boldsymbol{w}_{\boldsymbol{i}, \boldsymbol{j}}$ represents the time-averaged wind vector, which is obtained from the WRF-CMAQ driven by the European Centre for Medium-range Weather Forecasts (ECMWF) ERA5 dataset. $\boldsymbol{\nabla} \cdot\left(\boldsymbol{k}_{i, j} \cdot \boldsymbol{\nabla} \boldsymbol{V}_{i, j}\right)$ represents the diffusion term. Therein $\boldsymbol{k}_{i, j}$ is the diffusion coefficient tensor (in $\left.\mathrm{m}^{2} \cdot \mathrm{s}^{-1}\right)$, which indicates the diffusion transport driven by the residual winds. This process could be simplified as follows (Eqs. $2 \sim 4$ ):

$\boldsymbol{\nabla} \cdot\left(\boldsymbol{k}_{i, j} \cdot \boldsymbol{\nabla} \boldsymbol{V}_{i, j}\right)=\boldsymbol{\nabla} \cdot\left(\boldsymbol{k}_{i} \cdot \boldsymbol{\nabla} \boldsymbol{V}_{i}\right)+\boldsymbol{\nabla} \cdot\left(\boldsymbol{k}_{j} \cdot \boldsymbol{\nabla} \boldsymbol{V}_{j}\right)($ Eq. 2),

$\boldsymbol{k}_{i}=\frac{1}{2}\left(\overline{w_{l}}\right)^{2} t(\mathrm{Eq} .3)$

$\boldsymbol{k}_{j}=\frac{1}{2}\left(\overline{\boldsymbol{w}_{J}}\right)^{2} \boldsymbol{t}($ Eq. 4$)$.

Therein $\boldsymbol{k}_{\boldsymbol{i}}$ and $\boldsymbol{k}_{\boldsymbol{j}}$ are the diffusion coefficients in the zonal and meridional directions, respectively. We applied a random walk assumption to derive the diffusion coefficients. The random walk step was assumed to be fixed and equal to the deviation of wind speed in the zonal or meridional direction $\left(\overline{\boldsymbol{w}_{\boldsymbol{\imath}}} \boldsymbol{o r} \overline{\boldsymbol{w}_{\boldsymbol{J}}}\right) \cdot \boldsymbol{t}$ is one hour, compatible to the sampling temporal interval of the simulated wind information.

Note that a background column was first subtracted from the $\mathrm{NO}_{\mathrm{x}} \mathrm{VCDs}$, so as to include only the emission fluxes of the point sources responsible for the hotspots. The background column was estimated as the $5^{\text {th }}$ percentile of all the $1 \times 1 \mathrm{~km}^{2}$ oversampled grids ${ }^{7}$. The background correction is illustrated in the Supplementary Information.

Emissions of super-emitters and cities were also calculated in MEICv1.3 and used to compare with our satellite-based emission estimates. For 2016, this bottom-up inventory was established at a spatial resolution of $0.25^{\circ} \times 0.25^{\circ}$ and of five 


\section{Submitted to Nature Communication}

anthropogenic sectors (i.e., agriculture, power plant, industry, residential, and transportation). According to our superresolution results, the inventory was first re-gridded to the $1 \times 1 \mathrm{~km}^{2}$ resolution. For the cities, the emissions in MEICv1.3 were then summed over all sectors, while, for the super-emitters, only those of industry and power plant sectors were considered. Specifically, these super-emitters were attributed to industrial hotspots or parks, including the following sectors: power industry, oil refineries, transformation industry, combustion for manufacturing, and process emissions during production and application. Note that only the $1 \times 1 \mathrm{~km}^{2}$ grids that contained the super-emitters were used to calculate the averaged emission estimated.

For several reasons, MEICv1.3 may underestimate the emissions of the super-emitters even more than shown in Fig. 3. First, the main reason, as explained above, is attributed to the $\mathrm{NO}_{\mathrm{x}}$ lifetime, which could be smaller than the 4 hours we assumed, directly resulting in an underestimation of our estimates. Second, depending on the thermal contrast, the TROPOMI instrument, like any other infrared instrument, can miss the lower layer of the atmosphere in which $\mathrm{NO}_{\mathrm{x}}$ is emitted. Hence, our results are more likely to underestimate $\mathrm{NO}_{\mathrm{x}} \mathrm{VCDs}$ than to overestimate them.

\section{Identification and attribution}

By combining the tropospheric $\mathrm{NO}_{2} \mathrm{VCDs}$, the top-down $\mathrm{NO}_{\mathrm{x}}$ emission estimates, and the high-quality visible imageries from Landsat 8, we identified the super-emitters manually. Although automated ways with uniform thresholds might result in more consistent and flexible identifications, no satisfactory set of criteria was found that could be applied for the superemitters nationwide. This is mainly because, in localized regions, the $\mathrm{NO}_{\mathrm{x}}$ budgets respond to the changes in not only the super-emitters but also the background. In addition, the wildfire emergencies generally emitted elevated $\mathrm{NO}_{\mathrm{x}}$ plumes and especially hamper the identification of hotspots over a large area, even on a one-year average (for example, most of Northeast and Southwest China) ${ }^{46}$. We thus combined the MODIS fire product and the high-quality visible imageries to eliminate the fire hotspots.

To identify the super-emitters manually, we identified the localized maxima of both VCDs and emissions, which was significantly higher than the background value. Along with the visible imageries, we could attribute the characteristic enhancement to an industrial hotspot or park. As a result, the typical area with a super-emitter could be identified with a limited geographical scope (i.e., $<5 \times 5 \mathrm{~km}^{2}$ ). Each super-emitter was approximated as a rectangle on the latitude-longitude grid, the central, minimum, and maximum coordinates of which were recorded in Supplementary Table 1. We must highlight that, within densely source areas (e.g., megacities), the emission gradients from numerous super-emitters interfered with each other and were inevitably missed.

Although the visible imageries enabled us to ascertain the locations of the super-emitters, it was not possible to identify the industry type directly. Particularly, in China, where the industry is still rapidly developing, the associated bottom-up information may not be assessible. In addition, the Baidu Map allowed us to assign a name to each super-emitter. The usual choice was the name of the specific address recorded in Baidu Map or the name of the nearest geographical area. 


\section{Reference}

1. Richter, A., Burrows, J. P., Nüß, H., Granier, C. \& Niemeier, U. Increase in tropospheric nitrogen dioxide over China observed from space. Nature 437, 129-132 (2005).

2. Edwards, P. M. et al. High winter ozone pollution from carbonyl photolysis in an oil and gas basin. Nature 514, 351-354 (2014).

3. Wang, J. et al. Fast sulfate formation from oxidation of $\mathrm{SO}_{2}$ by $\mathrm{NO}_{2}$ and $\mathrm{HONO}$ observed in Beijing haze. Nat. Commun. 11, 2844 (2020).

4. Zhang, R. et al. Formation of urban fine particulate matter. Chem. Rev. 115, 3803-3855 (2015).

5. Martin, R. V et al. Global inventory of nitrogen oxide emissions constrained by space-based observations of $\mathrm{NO}_{2}$ columns. J. Geophys. Res. Atmos. 108, (2003).

6. Beirle, S., Boersma, K. F., Platt, U., Lawrence, M. G. \& Wagner, T. Megacity emissions and lifetimes of nitrogen oxides probed from space. Science. 333, 1737-1739 (2011).

. Beirle, S. et al. Pinpointing nitrogen oxide emissions from space. Sci. Adv. 5, eaax9800 (2019).

8. Tong, D. et al. Targeted emission reductions from global super-polluting power plant units. Nat. Sustain. 1, 59-68 (2018).

9. Tang, L. et al. Substantial emission reductions from Chinese power plants after the introduction of ultra-low emissions standards. Nat. Energy 4, 929-938 (2019).

10. Zheng, H. et al. Development of a unit-based industrial emission inventory in the Beijing-Tianjin-Hebei region and resulting improvement in air quality modeling. Atmos. Chem. Phys. 19, 3447-3462 (2019).

11. China, S. C. of the P. R. of. Air pollution prevention and control action plan. http://www.gov.cn/zwgk/201309/12/content_2486773.htm (2013).

12. The Ministry of Ecology and Environment of China. Action plan for comprehensive control of air pollution in Beijing-Tianjin-Hebei and surrounding areas in autumn and winter from 2017 to 2018. 7-143 (2017).

13. Griffin, D. et al. High-resolution mapping of nitrogen dioxide with TROPOMI: First results and validation over the Canadian oil sands. Geophys. Res. Lett. 46, 1049-1060 (2019).

14. Kong, H. et al. High-resolution $\left(0.05^{\circ} \times 0.05^{\circ}\right) \mathrm{NO}_{\mathrm{x}}$ emissions in the Yangtze River Delta inferred from OMI. Atmos. Chem. Phys. 19, 12835-12856 (2019).

15. Li, M. et al. MIX: a mosaic Asian anthropogenic emission inventory under the international collaboration framework of the MICS-Asia and HTAP. Atmos. Chem. Phys. 17, (2017).

16. Janssens-Maenhout, G. et al. HTAP_v2. 2: a mosaic of regional and global emission grid maps for 2008 and 2010 to study hemispheric transport of air pollution. Atmos. Chem. Phys. 15, 11411-11432 (2015).

17. McLinden, C. A. et al. Space-based detection of missing sulfur dioxide sources of global air pollution. Nat. Geosci. 9 , 496-500 (2016). 
373 18. Van Damme, M. et al. Industrial and agricultural ammonia point sources exposed. Nature 564, 99-103 (2018).

374 19. Tang, L. et al. Air pollution emissions from Chinese power plants based on the continuous emission monitoring systems network. Sci. Data 7, 325 (2020).

20. Zhang, Q. et al. $\mathrm{NO}_{\mathrm{x}}$ emission trends for China, 1995-2004: The view from the ground and the view from space. $J$. Geophys. Res. Atmos. 112, (2007).

378 21. Levelt, P. F. et al. The ozone monitoring instrument. IEEE Trans. Geosci. Remote Sens. 44, 1093-1101 (2006).

22. Ding, J. et al. Chinese $\mathrm{NO}_{\mathrm{x}}$ emission reductions and rebound as a result of the COVID-19 crisis quantified through inversion of TROPOMI NO${ }_{2}$ observations. (2020). doi:10.1002/essoar.10503145.1.

23. Liu, F. et al. $\mathrm{NO}_{\mathrm{x}}$ emission trends over Chinese cities estimated from OMI observations during 2005 to 2015. Atmos. Chem. Phys. 17, 9261-9275 (2017).

24. Liu, J. et al. Air pollutant emissions from Chinese households: A major and underappreciated ambient pollution source. Proc. Natl. Acad. Sci. 113, 7756-7761 (2016).

25. Tao, S. et al. Quantifying the rural residential energy transition in China from 1992 to 2012 through a representative national survey. Nat. Energy 3, 567-573 (2018).

26. Zhang, Q. et al. Drivers of improved $\mathrm{PM}_{2.5}$ air quality in China from 2013 to 2017. Proc. Natl. Acad. Sci. (2019). autumn and winter of 2020-2021 in Beijing-Tianjin-Hebei and its surrounding areas and the Fenwei plain. https://www.mee.gov.cn/xxgk2018/xxgk/xxgk03/202011/t20201103_806152.html (2020).

28. Guo, Y., Tian, J. \& Chen, L. Managing energy infrastructure to decarbonize industrial parks in China. Nat. Commun. 11, 981 (2020).

29. Mijling, B. \& R., J. van der A. Using daily satellite observations to estimate emissions of short-lived air pollutants on a mesoscopic scale. J. Geophys. Res. Atmos. 117, (2012).

30. Reichstein, M. et al. Deep learning and process understanding for data-driven Earth system science. Nature 566, 195-204 (2019).

31. Pandey, S. et al. Satellite observations reveal extreme methane leakage from a natural gas well blowout. Proc. Natl. Acad. Sci. 116, 26376 - 26381 (2019).

32. Varon, D. J., Jacob, D. J., Jervis, D. \& McKeever, J. Quantifying Time-Averaged Methane Emissions from Individual Coal Mine Vents with GHGSat-D Satellite Observations. Environ. Sci. Technol. 54, 10246-10253 (2020).

33. Varon, D. et al. Satellite Discovery of Anomalously Large Methane Point Sources From Oil/Gas Production. Geophys. Res. Lett. 46, (2019).

34. Goldberg, D. L. et al. Enhanced Capabilities of TROPOMI NO 2 : Estimating $\mathrm{NO}_{\mathrm{X}}$ from North American Cities and Power Plants. Environ. Sci. Technol. 53, 12594-12601 (2019). 
35. TROPOMI ATBD of the total and tropospheric NO2 data products.

https://sentinel.esa.int/documents/247904/2476257/Sentinel-5P-TROPOMI-ATBD-NO2-data-products.

36. TROPOMI ATBD of the total and tropospheric NO2 data products.

37. Wong, D. C. et al. WRF-CMAQ two-way coupled system with aerosol feedback: software development and preliminary results. Geosci. Model Dev. 5, 299-312 (2012).

38. Yu, S. et al. Aerosol indirect effect on the grid-scale clouds in the two-way coupled WRF-CMAQ: model description, development, evaluation and regional analysis. Atmos. Chem. Phys. Discuss. 25649 (2013).

39. Wang, L. et al. Significant wintertime PM\$_Imathbf $\{2.5\} \$$ mitigation in the Yangtze River Delta, China from 2016 to 2019: observational constraints on anthropogenic emission controls. Atmos. Chem. Phys. Discuss. 2020, 1-31 (2020).

40. Mehmood, K. et al. Relative effects of open biomass burning and open crop straw burning on haze formation over central and eastern China: modeling study driven by constrained emissions. Atmos. Chem. Phys. 20, 2419-2443 (2020).

41. Li, X. et al. The impact of observation nudging on simulated meteorology and ozone concentrations during DISCOVER-AQ 2013 Texas campaign. Atmos. Chem. Phys 16, 3127-3144 (2016).

42. Sun, K. et al. A physics-based approach to oversample multi-satellite, multispecies observations to a common grid. Atmos. Meas. Tech. 11, 6679-6701 (2018).

43. Fioletov, V. E., McLinden, C. A., Krotkov, N., Moran, M. D. \& Yang, K. Estimation of SO2 emissions using OMI retrievals. Geophys. Res. Lett. 38, (2011).

44. Laughner, J. L. \& Cohen, R. C. Direct observation of changing $\mathrm{NO}_{\mathrm{x}}$ lifetime in North American cities. Science. 366, 723-727 (2019).

45. Wolfe, G. M. et al. Mapping hydroxyl variability throughout the global remote troposphere via synthesis of airborne and satellite formaldehyde observations. Proc. Natl. Acad. Sci. 116, 11171-11180 (2019).

46. Mehmood, K. et al. Relative effects of open biomass burning and open crop straw burning on haze formation over central and eastern China: modeling study driven by constrained emissions. Atmos. Chem. Phys. 20, 2419-2443 (2020).

\section{Data availability.}

The TROPOMI data is available in https://scihub.copernicus.eu/. The DECSO-based $\mathrm{NO}_{\mathrm{x}}$ emission is obtained from https://www.temis.nl/emissions/data.php. The MEICv1.3 is from http://meicmodel.org/. The ERA5 meteorological reanalysis dataset comes from https://www.ecmwf.int/en/forecasts/datasets/reanalysis-datasets/era5. The Landsat 8 data is available in https://developers.google.com/earth-engine/datasets/catalog/landsat-8. Additional data related to this paper may be requested from the authors. 


\section{Supplementary materials.}

438 Supplementary information is available online.

\section{Author contributions.}

440 P.L. and C. W. designed this study and wrote the manuscript. P.L. and Y.P. developed the retrieval algorithm. P.L., P. S., 441 L.D., M. L., N. X., J. L., S. L., and Y.L. derived the hyperfine maps and performed the analysis. C. W., J. M., X. Z., W. W., 442 Y. S., W. S., G. W., L. W., X. C., Y. X., L. J., Y. Z., M. L., Z. L., and W. L. contributed to the discussions. S. Y., R. D., and 443 J.H.S contributed to the manuscript and supervised the research.

\section{Competing interests.}

445 The authors declare no competing interests.

\section{Acknowledgements.}

447 We thank ESA and the S-5P/TROPOMI level 1 and level 2 teams for the great work on initiating and realizing TROPOMI 448 data. This study is supported by National Natural Science Foundation of China (No. 22006030, 22076172, 21577126 and 449 41561144004), Hebei Youth Top Fund (BJ2020032), Research Fund of Education Bureau of Hebei (QN2019184), Initiation 450 Fund of Hebei Agricultural University (412201904 and YJ201833), the Department of Science and Technology of China 451 (No. 2016YFC0202702, 2018YFC0213506 and 2018YFC0213503), National Research Program for Key Issues in Air 452 Pollution Control in China (No. DQGG0107). 